\title{
Orbital magnetic susceptibility of finite-sized graphene
}

\author{
Yuya Ominato and Mikito Koshino \\ Department of Physics, Tohoku University, Sendai 980-8578, Japan
}

(Dated: September 4, 2018)

\begin{abstract}
We study the orbital magnetism of graphene ribbon in the effective-mass approximation, to figure out the finite-size effect on the singular susceptibility known in the bulk limit. We find that the susceptibility at $T=0$ oscillates between diamagnetism and paramagnetism as a function of $\varepsilon_{F}$, in accordance with the subband structure formed by quantum confinement. In increasing $T$, the oscillation rapidly disappears once the thermal broadening energy exceeds the subband spacing, and the susceptibility $\chi\left(\varepsilon_{F}\right)$ approaches the bulk limit i.e., a thermally broadened diamagnetic peak centered at $\varepsilon_{F}=0$. The electric current supporting the diamagnetism is found to flow near the edge with a depth $\sim \hbar v /\left(2 \pi k_{B} T\right)$, with $v$ being the band velocity, while at $T=0$ the current distribution spreads entirely in the sample reflecting the absence of the characteristic wavelength in graphene. The result is applied to estimate the three-dimensional random-stacked multilayer graphene, where we show that the external magnetic field is significantly screened inside the sample in low temperatures, in a much stronger manner than in graphite.
\end{abstract}

\section{INTRODUCTION}

Graphite is known as one of the strongest diamagnetic materials among natural substances. ${ }^{1-3}$ This property is due to the large orbital diamagnetism related to the small effective mass in the band structure, i.e., narrow energy gap between conduction and valence bands. The diamagnetic effect becomes even greater in graphene monolayer $^{4-6}$ which is truly a zero-gap system. ${ }^{1,7-9}$ The magnetic susceptibility of graphene at zero temperature contains a singularity expressed as a delta function in Fermi energy $\varepsilon_{F}$, which diverges at Dirac point $\left(\varepsilon_{F}=0\right)$ where the two bands stick, and vanishes otherwise. ${ }^{1,10-17}$ The orbital diamagnetism has been studied for other graphene-related materials, such as graphite intercalation compounds, ${ }^{18,20-22}$ carbon nanotube, ${ }^{23-25}$ few-layer graphenes, ${ }^{19,26,27}$ and an organic material having similar gapless spectrum. ${ }^{28}$

In this paper, we investigate the orbital diamagnetism of a graphene strip with finite width. ${ }^{29-41}$ The purpose of this work is two-fold: (i) To understand how the deltafunction singularity of the bulk limit is relaxed in a realistic finite-sized graphene system. In the literatures, the orbital susceptibility of graphene nanoribbons was calculated for the Fermi energies near the Dirac point, ${ }^{31,44}$ while the behavior off Dirac point and the relation to the bulk susceptibility is not well understood. (ii) To study the diamagnetic current flow on graphene. In the conventional diamagnetism of metal, we usually expect that the current circulates near the surface with a depth of the order of the Fermi wave length $\lambda_{F}$. In graphene, the only characteristic length scale $\lambda_{F}$ intrinsically diverges, and we expect a peculiar current distribution different from the conventional system.

To address above problems, here we calculate the orbital susceptibility and the current distribution of graphene ribbon with an arbitrary width, in various Fermi energies $\varepsilon_{F}$ and temperatures $T$, using the effective mass approximation. We find that the susceptibility at $T=0$ oscillates between diamagnetic and paramagnetic values in increasing $\varepsilon_{F}$, in accordance with the detailed subband structure. In increasing temperature, the oscillation rapidly disappears once the thermal broadening energy exceeds the subband spacing, and the susceptibility approaches bulk limit, i.e., a thermally-broadened diamagnetic peak centered at $\varepsilon_{F}=0$, independently of the atomic configuration at the edge. We also apply a similar analysis to the carbon nanotube, and find a similar oscillation in the susceptibility.

The electric current supporting the diamagnetism spreads entirely in the sample at $T=0$, reflecting the absence of the characteristic wavelength. In increasing temperature, however, the current density tends to localize near the boundary with a depth $\sim \hbar v /\left(2 \pi k_{B} T\right)$, forming an edge current circulation.

The analysis of the spatial distribution of the diamagnetic current is useful in studying a graphene stack where the diamagnetic current of one layer influences the electron motion in other layers. If we take a randomlystacked graphene multilayer, in which the interlayer coupling is expected to be small, ${ }^{45-51}$ the self-consistent calculation shows that the diamagnetism is much stronger than in graphite, and the external magnetic field is significantly screened inside the sample in low temperatures.

The paper is organized as follows. In Sec. II, we briefly review effective mass description of electrons in graphene ribbon, and formulate the orbital magnetic susceptibility. We present the numerical results and detailed discussion in Sec. III, as well as a similar analysis for the carbon nanotube in Sec. IV. We argue the diamagnetism of randomly stacked graphene multilayers in Sec. V. The conclusion is given in Sec. VI.

\section{FORMULATIONS}

\section{A. Effective mass approximation}

Graphene is composed of a honeycomb network of carbon atoms, where a unit cell contains a pair of sublattices, 
(a) Zigzag ribbon

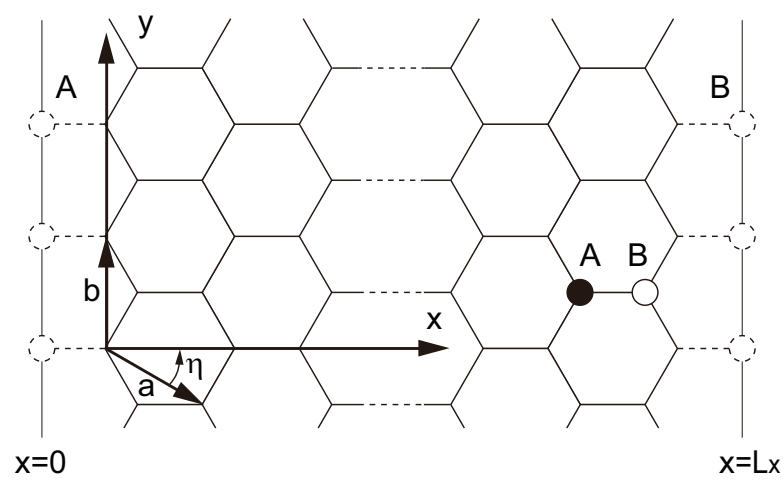

(b) Armchair ribbon

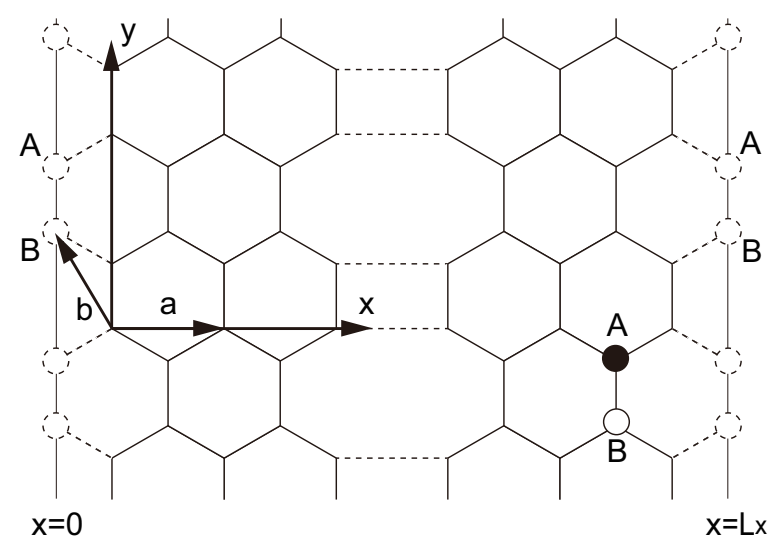

FIG. 1: Atomic structures of graphene ribbons with (a) zigzag boundary and (b) armchair boundary, respectively. Dashed circles indicate missing sites beyond the boundary.

denoted by $A$ and $B$. Fig.1 (a) and (b) show the atomic structure of zigzag and armchair graphene ribbons, respectively, where $\boldsymbol{a}$ and $\boldsymbol{b}$ are primitive translation vectors of infinite graphene. The lattice constant is given by $a=|\boldsymbol{a}| \approx 0.246 \mathrm{~nm}$. For both cases we set $y$-axis along the ribbon, and set $x=0$ and $L_{x}$ to the line of missing sites nearest from the edge. We define $\eta$ as the angle between $x$ axis and $\boldsymbol{a}$, which is $\pi / 6$ for zigzag, and 0 for armchair boundary.

In a tight-binding model, the wave function of graphene electron is written as

$\psi(\boldsymbol{r})=\sum_{\boldsymbol{R}_{\mathrm{A}}} \psi_{\mathrm{A}}\left(\boldsymbol{R}_{\mathrm{A}}\right) \phi\left(\boldsymbol{r}-\boldsymbol{R}_{\mathrm{A}}\right)+\sum_{\boldsymbol{R}_{\mathrm{B}}} \psi_{\mathrm{B}}\left(\boldsymbol{R}_{\mathrm{B}}\right) \phi\left(\boldsymbol{r}-\boldsymbol{R}_{\mathrm{B}}\right)$,

where $\boldsymbol{R}_{\mathrm{A}}$ and $\boldsymbol{R}_{\mathrm{B}}$ are the positions of A-sites and Bsites, respectively, and $\phi(\boldsymbol{r})$ denotes the wave function of the $p_{z}$ orbital of a carbon atom.

For states in the vicinity of the Fermi level $\varepsilon=0$, the wave amplitudes are written $\operatorname{as}^{53}$

$$
\begin{aligned}
& \psi_{\mathrm{A}}\left(\boldsymbol{R}_{\mathrm{A}}\right)=e^{i \boldsymbol{K} \cdot \boldsymbol{R}_{\mathrm{A}}} F_{\mathrm{A}}^{\mathrm{K}}\left(\boldsymbol{R}_{\mathrm{A}}\right)+e^{i \eta} e^{i \boldsymbol{K}^{\prime} \cdot \boldsymbol{R}_{\mathrm{A}}} F_{\mathrm{A}}^{\mathrm{K}^{\prime}}\left(\boldsymbol{R}_{\mathrm{A}}\right), \\
& \psi_{\mathrm{B}}\left(\boldsymbol{R}_{\mathrm{B}}\right)=-\omega e^{i \eta} e^{i \boldsymbol{K} \cdot \boldsymbol{R}_{\mathrm{B}}} F_{\mathrm{B}}^{\mathrm{K}}\left(\boldsymbol{R}_{\mathrm{B}}\right)+e^{i \boldsymbol{K}^{\prime} \cdot \boldsymbol{R}_{\mathrm{B}}} F_{\mathrm{B}}^{\mathrm{K}^{\prime}}\left(\boldsymbol{R}_{\mathrm{B}}\right) .
\end{aligned}
$$

in terms of the slowly-varying envelope functions $F_{\mathrm{A}}^{\mathrm{K}}, F_{\mathrm{B}}^{\mathrm{K}}, F_{\mathrm{A}}^{\mathrm{K}^{\prime}}$, and $F_{\mathrm{B}}^{\mathrm{K}^{\prime}}$. The envelope functions satisfy the Schrödinger equation, ${ }^{1,7-9,52,53}$

$$
\mathcal{H}_{0} \boldsymbol{F}(\boldsymbol{r})=\varepsilon \boldsymbol{F}(\boldsymbol{r})
$$

with

$$
\begin{gathered}
\mathcal{H}_{0}=\hbar v\left(\begin{array}{cccc}
0 & \hat{k}_{x}-i \hat{k}_{y} & 0 & 0 \\
\hat{k}_{x}+i \hat{k}_{y} & 0 & 0 & 0 \\
0 & 0 & 0 & \hat{k}_{x}+i \hat{k}_{y} \\
0 & 0 & \hat{k}_{x}-i \hat{k}_{y} & 0
\end{array}\right), \\
\boldsymbol{F}(\boldsymbol{r})=\left(\begin{array}{c}
F_{\mathrm{A}}^{\mathrm{K}}(\boldsymbol{r}) \\
F_{\mathrm{B}}^{\mathrm{K}}(\boldsymbol{r}) \\
F_{\mathrm{A}}^{\mathrm{K}^{\prime}}(\boldsymbol{r}) \\
F_{\mathrm{B}}^{\mathrm{K}^{\prime}}(\boldsymbol{r})
\end{array}\right),
\end{gathered}
$$

where $\hat{\boldsymbol{k}}=-i \boldsymbol{\nabla}$ and $v$ is the band velocity.

The electronic states of the graphene ribbon can be correctly described by setting the appropriate boundary condition to the effective mass Hamiltonian. ${ }^{34}$ Now the eigenstates are labeled by $k_{y}$ since the system is translationally symmetric along $y$. A wave function of $k_{y}$ and the energy $\varepsilon$ is generally written as

$$
\boldsymbol{F}(\boldsymbol{r})=e^{i k_{y} y}\left(\begin{array}{c}
A e^{i k_{x} x}+B e^{-i k_{x} x} \\
s\left(A e^{i\left(k_{x} x+\theta\right)}-B e^{-i\left(k_{x} x+\theta\right)}\right) \\
C e^{i k_{x} x}+D e^{-i k_{x} x} \\
s\left(C e^{i\left(k_{x} x-\theta\right)}-D e^{-i\left(k_{x} x-\theta\right)}\right)
\end{array}\right),
$$

where $k_{x}^{2}=\varepsilon^{2} / \hbar^{2} v^{2}-k_{y}^{2}, e^{i \theta}=\left(k_{x}+i k_{y}\right) / \sqrt{k_{x}^{2}+k_{y}^{2}}, s=$ $\varepsilon /|\varepsilon|$, and $A, B, C$ and $D$ are numbers to be determined by satisfying the boundary condition, as we will argue in the following.

\section{B. Zigzag boundary}

In the zigzag ribbon, the boundary condition is given by $\psi_{\mathrm{A}}\left(\boldsymbol{R}_{\mathrm{A}}\right)=0$ at $x=0$, and $\psi_{\mathrm{B}}\left(\boldsymbol{R}_{\mathrm{B}}\right)=0$ at $x=L_{x}$. By using Eq. (2), this is translated to the condition for the envelope function as

$$
\begin{aligned}
F_{\mathrm{A}}^{\mathrm{K}}(0, y) & =0, \\
F_{\mathrm{B}}^{\mathrm{K}}\left(L_{x}, y\right) & =0, \\
F_{\mathrm{A}}^{\mathrm{K}^{\prime}}(0, y) & =0, \\
F_{\mathrm{B}}^{\mathrm{K}^{\prime}}\left(L_{x}, y\right) & =0,
\end{aligned}
$$




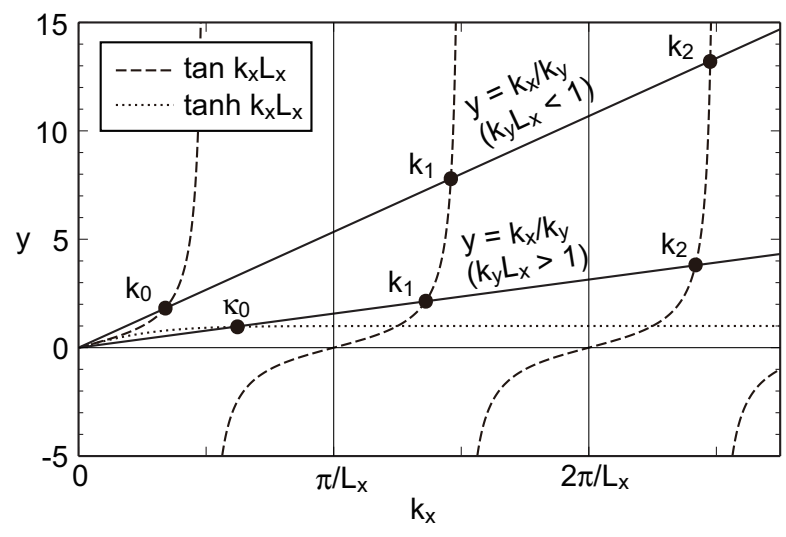

FIG. 2: Plots to find solutions of Eq.(9) and Eq.(11).

which keeps the states at $\mathrm{K}$ and those at $\mathrm{K}^{\prime}$ independent. For an eigenstate for the $\mathrm{K}$ point, we apply the first two lines of Eq.(7) to Eq.(6), to obtain

$$
\begin{aligned}
& A+B=0, \\
& s\left(A e^{i\left(k_{x} L_{x}+\theta\right)}-B e^{-i\left(k_{x} L_{x}+\theta\right)}\right)=0 .
\end{aligned}
$$

To have a solution other than $A=B=0$, we require ${ }^{34}$

$$
k_{y}=\frac{k_{x}}{\tan k_{x} L_{x}} .
$$

For given $k_{y}$, we define $k_{n}(n=0,1,2, \cdots)$ as solution of Eq. (9) in $k_{x}$ satisfying $n \pi<k_{n} L_{x}<(n+1) \pi$. Corresponding eigenstates and the energy are obtained as

$$
\begin{aligned}
& \boldsymbol{F}_{s n k_{y}}(\boldsymbol{r})=A_{n} \frac{e^{i k_{y} y}}{\sqrt{L_{x} L_{y}}}\left(\begin{array}{c}
i \sin k_{n} x \\
s(-1)^{n+1} \sin k_{n}\left(x-L_{x}\right) \\
0 \\
0
\end{array}\right) \\
& A_{n}=\left(1-\frac{\sin 2 k_{n} L_{x}}{2 k_{n} L_{x}}\right)^{-1 / 2} \\
& \varepsilon_{s n k_{y}}=s \hbar v \sqrt{k_{n}^{2}+k_{y}^{2}}
\end{aligned}
$$

for $n=0,1,2, \cdots$. For the normalization of the wavefunction, we assumed the periodic boundary condition in $y$-direction with a large enough period $L_{y}$.

Solution $k_{n}$ is obtained by searching for crossing points of $\tan k_{x} L_{x}$ and $k_{x} / k_{y}$ as illustrated in Fig. 2. When $k_{y} L_{x}>1$, the first solution $k_{0}$ becomes a pure imaginary number $i \kappa_{0}$ which satisfies

$$
k_{y}=\frac{\kappa_{0}}{\tanh \kappa_{0} L_{x}} .
$$

The wavefunction and the energy then becomes

$$
\begin{aligned}
& \boldsymbol{F}_{s 0 k_{y}}(\boldsymbol{r})=A_{1} \frac{e^{i k_{y} y}}{\sqrt{L_{x} L_{y}}}\left(\begin{array}{c}
i \sinh \kappa_{0} x \\
-s \sinh \kappa_{0}\left(x-L_{x}\right) \\
0 \\
0
\end{array}\right), \\
& A_{0}=\left(-1+\frac{\sinh 2 \kappa_{0} L_{x}}{2 \kappa_{0} L_{x}}\right)^{-1 / 2}, \\
& \varepsilon_{s 0 k_{y}}=s \hbar v \sqrt{-\kappa_{0}^{2}+k_{y}^{2}} .
\end{aligned}
$$

This actually describes the edge state localized at the boundary $x=0$ and $L_{x}$ giving a nearly flat energy band. ${ }^{29-31}$

The eigenenergy $\varepsilon_{s n k_{y}}$ represents the $n$-th branch of conduction $(s=+)$ and valence $(s=-)$ bands respectively. Energy band structure of $\mathrm{K}$ as a function of $k_{y}$ is shown as solid curves in Fig.3(a). Eigenstates for $\mathrm{K}^{\prime}$ point are obtained similarly, where the energy band structure is equivalent to Fig. 3(a) with $k_{y}$ inverted to $-k_{y}$. The flat band of edge states of $\mathrm{K}$ and $\mathrm{K}^{\prime}$ are connected in a wave number away from $\mathrm{K}$ or $\mathrm{K}^{\prime} .{ }^{29-31}$

\section{Armchair boundary}

In the armchair ribbon, the boundary condition imposes both of $\psi_{\mathrm{A}}\left(\boldsymbol{R}_{\mathrm{A}}\right)=0$ and $\psi_{\mathrm{B}}\left(\boldsymbol{R}_{\mathrm{B}}\right)=0$ at each of $x=0$ and $x=L_{x}$. The corresponding conditions for the envelope functions are written as

$$
\begin{aligned}
F_{\mathrm{A}}^{\mathrm{K}}(0, y)+F_{\mathrm{A}}^{\mathrm{K}^{\prime}}(0, y) & =0, \\
F_{\mathrm{B}}^{\mathrm{K}}(0, y)-F_{\mathrm{B}}^{\mathrm{K}^{\prime}}(0, y) & =0, \\
F_{\mathrm{A}}^{\mathrm{K}}\left(L_{x}, y\right)+\omega^{-2 N} F_{\mathrm{A}}^{\mathrm{K}^{\prime}}\left(L_{x}, y\right) & =0, \\
F_{\mathrm{B}}^{\mathrm{K}}\left(L_{x}, y\right)-\omega^{-2 N} F_{\mathrm{B}}^{\mathrm{K}^{\prime}}\left(L_{x}, y\right) & =0,
\end{aligned}
$$

where $N=L_{x} / a$ is the number of honeycomb lattices between $x=0$ and $L_{x}$, which can be integer or halfinteger depending on the position of the edge.

Applying above conditions to Eq.(6), we obtain

$$
\left(\begin{array}{cccc}
1 & 1 & 1 & 1 \\
e^{i \theta} & -e^{-i \theta} & -e^{-i \theta} & e^{i \theta} \\
e^{i \lambda} & e^{-i \lambda} & \alpha e^{i \lambda} & \alpha e^{-i \lambda} \\
e^{i(\lambda+\theta)} & -e^{-i(\lambda+\theta)} & -\alpha e^{i(\lambda+\theta)} & \alpha e^{-i(\lambda+\theta)}
\end{array}\right)\left(\begin{array}{l}
A \\
B \\
C \\
D
\end{array}\right)=\left(\begin{array}{l}
0 \\
0 \\
0 \\
0
\end{array}\right),
$$

where $\alpha=\omega^{-2 N}$ and $\lambda=k_{x} L_{x}$. The determinant of matrix in Eq.(14) should vanish to have a non-zero solution. This condition is reduced to

$$
k_{x}=k_{n} \equiv \frac{\pi}{L_{x}}\left(n-\frac{\nu}{3}\right), n=0, \pm 1, \pm 2, \cdots,
$$

$\nu$ is an integer $(0, \pm 1)$ defined by

$$
2 N=3 m+\nu,
$$


with integer $m$. The eigenstate and energy are obtained as

$$
\begin{aligned}
& \boldsymbol{F}_{s n k_{y}}(\boldsymbol{r})=\frac{e^{i k_{y} y}}{2 \sqrt{L_{x} L_{y}}}\left(\begin{array}{c}
e^{i k_{n} x} \\
s e^{i\left(k_{n} x+\theta\right)} \\
-e^{-i k_{n} x} \\
s e^{-i\left(k_{n} x-\theta\right)}
\end{array}\right), \\
& \varepsilon_{s n k_{y}}=s \hbar v \sqrt{k_{n}^{2}+k_{y}^{2}} .
\end{aligned}
$$

When $\nu=0$, the energy bands of $n=0$ and $s= \pm$ stick together and thus the system is metallic, while otherwise a gap opens at zero energy and the system becomes a semiconductor. Energy bands for metallic armchair ribbon $(\nu=0)$ and semiconducting armchair ribbon $(\nu= \pm 1)$ are shown in Fig. 3(b) and (c), respectively. In (c), the labeling $n$ is for the case of $\nu=+1$, while $n$ becomes $-n$ in $\nu=-1$.

\section{Orbital susceptibility}

To calculate the orbital diamagnetism, we consider a graphene ribbon under a uniform magnetic field $B$ perpendicular to graphene plane. We take the Landau gauge and set the vector potential as

$$
\boldsymbol{A}(\boldsymbol{r})=\left[0, B\left(x-\frac{L_{x}}{2}\right)\right]
$$

The Hamiltonian in presence of the magnetic field is obtained by replacing $\hat{\boldsymbol{k}}$ by $\hat{\boldsymbol{k}}+e \boldsymbol{A} /(\hbar c)$, as

$$
\mathcal{H}=\mathcal{H}_{0}+\delta \mathcal{H}, \quad \delta \mathcal{H}=\frac{e}{c} \hat{y_{y}} A_{y}
$$

where $c$ is the light velocity, and

$$
\hat{v}_{y}=\frac{1}{\hbar} \frac{\partial \mathcal{H}}{\partial \hat{k}_{y}}=v\left(\begin{array}{cccc}
0 & -i & 0 & 0 \\
i & 0 & 0 & 0 \\
0 & 0 & 0 & i \\
0 & 0 & -i & 0
\end{array}\right)
$$

The operator of the electric current density is given by

$$
\hat{j}_{y}(\boldsymbol{r})=-\frac{e}{2}\left\{\hat{v}_{y} \delta\left(\boldsymbol{r}-\boldsymbol{r}^{\prime}\right)+\delta\left(\boldsymbol{r}-\boldsymbol{r}^{\prime}\right) \hat{v}_{y}\right\} .
$$

In the first order perturbation in $\delta \mathcal{H}$, the expectation value of the current density is written as

$$
j_{y}(\boldsymbol{r})=\sum_{\alpha} f\left(\varepsilon_{\alpha}\right) \sum_{\beta(\neq \alpha)} \frac{2 \operatorname{Re}\left[(\delta \mathcal{H})_{\alpha \beta}\left(\hat{j}_{y}(\boldsymbol{r})\right)_{\beta \alpha}\right]}{\varepsilon_{\alpha}-\varepsilon_{\beta}}
$$

where $\alpha$ and $\beta$ represent the unperturbed eigenstates of graphene ribbon, and $f(\varepsilon)=1 /\left[1+e^{\beta(\varepsilon-\mu)}\right]$ is the Fermi distribution function with the chemical potential $\mu$.

In a zigzag ribbon, the current density always vanishes at the edges $x=0$ and $L_{x}$, while it is not generally the case in armchair ribbons. This is obvious from at the matrix element of $\hat{j}_{y}$ between two eigen states $\boldsymbol{F}$ and $\boldsymbol{F}^{\prime}$,

$$
\left\langle\boldsymbol{F}^{\prime}\left|\hat{j}_{y}(\boldsymbol{r})\right| \boldsymbol{F}\right\rangle=i e v\left[F_{A}^{\prime K}(\boldsymbol{r})^{*} F_{B}^{K}(\boldsymbol{r})-F_{B}^{\prime K}(\boldsymbol{r})^{*} F_{A}^{K}(\boldsymbol{r})\right] .
$$

In the wavefunction of zigzag ribbon, Eqs. (10) and (12), the component $F_{A}^{K}$ is zero at $x=0$, and $F_{B}^{K}$ is at $L_{x}$, so that Eq. (23) vanishes at the both edges.

The current density on $x y$-plane is related to the local magnetic moment $m(\boldsymbol{r})$ in $z$-direction by

$$
j_{x}=c \frac{\partial m}{\partial y}, j_{y}=-c \frac{\partial m}{\partial x} .
$$

In the present case, $m(\boldsymbol{r})$ depends only on $x$ so that the total magnetization per area is

$$
\begin{aligned}
M & =\frac{1}{L_{x} L_{y}} \int_{0}^{L_{x}} \int_{0}^{L_{y}} m(x) \mathrm{d} x \mathrm{~d} y \\
& =\frac{1}{c L_{x}} \int_{0}^{L_{x}}\left(x-\frac{L_{x}}{2}\right) j_{y}(x) \mathrm{d} x .
\end{aligned}
$$

The magnetic susceptibility is written as

$$
\chi=\lim _{B \rightarrow 0} \frac{M}{B}=\frac{2}{c L_{x} L_{y}} \sum_{\alpha} f\left(\varepsilon_{\alpha}\right) \sum_{\beta(\neq \alpha)} \frac{\left|(\delta \mathcal{H} / B)_{\alpha \beta}\right|^{2}}{\varepsilon_{\beta}-\varepsilon_{\alpha}} .
$$

We calculate Eqs. (22) and (26) numerically. As we have infinite energy bands below zero, we introduce a cut-off function $g\left(\varepsilon_{\alpha}\right)$ which smoothly vanishes $\left|\varepsilon_{\alpha}\right|>\varepsilon_{c}$. In the following, we take $\varepsilon_{c}=50 \varepsilon_{0}$ where

$$
\varepsilon_{0}=\frac{2 \pi \hbar v}{L_{x}}
$$

is the typical energy scale for the subband structure. The result is actually converging in the limit of large $\varepsilon_{c}$.

The susceptibility of the infinite bulk graphene at zero temperature is given by ${ }^{1,13,20}$

$$
\chi_{\mathrm{gr}}\left(\varepsilon_{F}\right)=-g_{v} g_{s} \frac{e^{2} v^{2}}{6 \pi c^{2}} \delta\left(\varepsilon_{F}\right),
$$

where $g_{v}=g_{s}=2$ are the valley and spin degeneracies, respectively. At finite temperature, it becomes

$$
\begin{aligned}
\chi_{\mathrm{gr}}(\mu ; T) & =\int_{-\infty}^{\infty} d \varepsilon\left(-\frac{\partial f(\varepsilon)}{\partial \varepsilon}\right) \chi_{\mathrm{gr}}(\varepsilon) \\
& =-g_{v} g_{s} \frac{e^{2} v^{2}}{24 \pi c^{2}} \frac{1}{k_{B} T \cosh \left[\mu /\left(2 k_{B} T\right)\right]} .
\end{aligned}
$$

In the graphene ribbon, the characteristic unit of the susceptibility can be chosen as

$$
\chi_{0}=g_{v} g_{s} \frac{e^{2} v^{2}}{6 \pi c^{2}} \frac{1}{\varepsilon_{0}} .
$$


(a) Zigzag ribbon

(b) Armchair ribbon $(v=0)$

(c) Armchair ribbon $(v= \pm 1)$

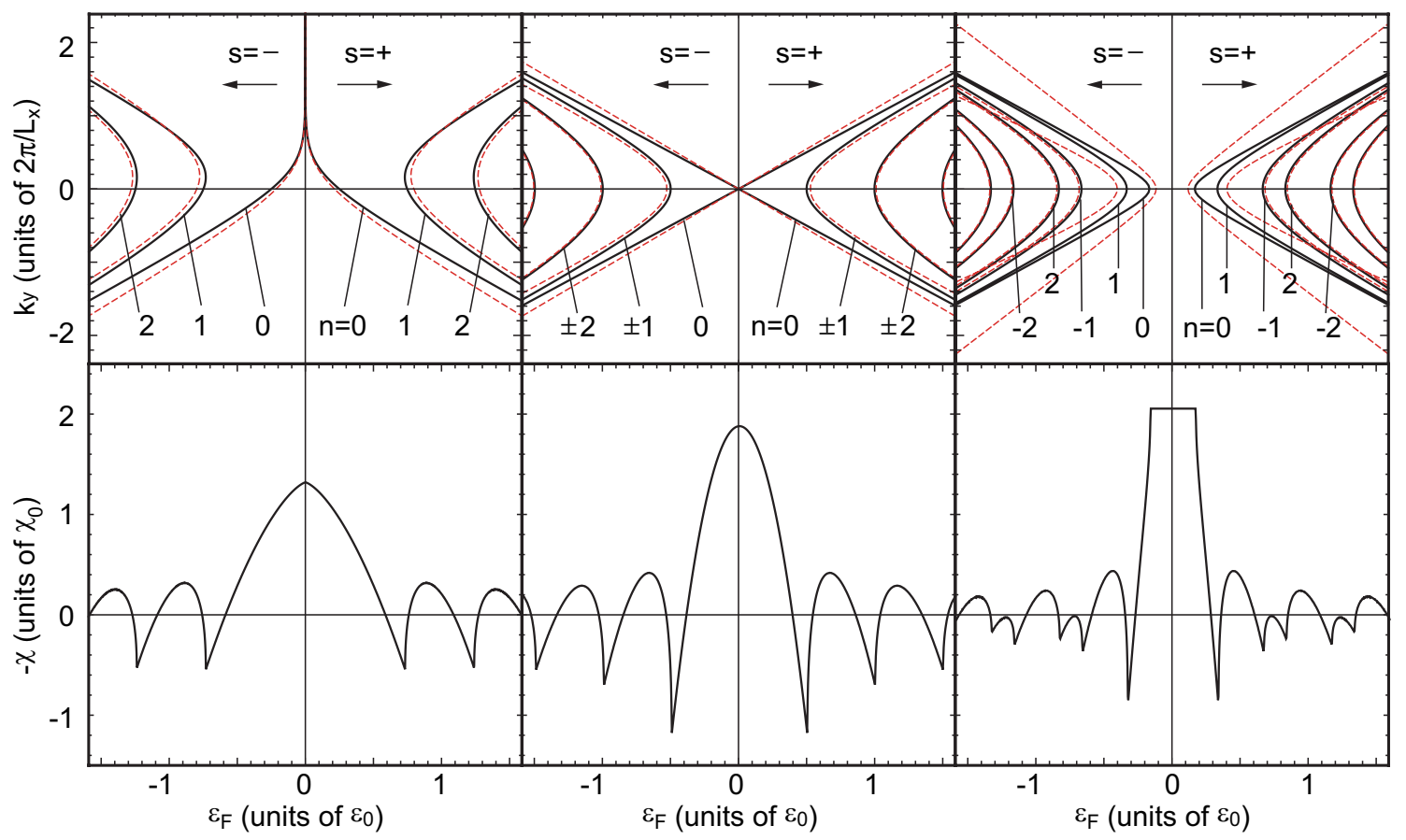

FIG. 3: Band structure (upper panel) and magnetic susceptibility as a function of $\varepsilon_{F}$ (lower), of (a) zigzag, (b) metallic armchair $(\nu=0)$ and (c) semiconducting armchair $(\nu= \pm 1)$ graphene ribbons. In upper panels, solid (black) and dashed (red) curves indicate the band structures at zero and a finite magnetic field, respectively. For the latter, the energy band is calculated with the perturbation theory in a magnetic field $B$. where we take $B=B_{0}$ for (a), and $B=0.5 B_{0}$ in (b) and (c) for illustrative purpose, and $B_{0}=(h / e) / L_{x}^{2}$.

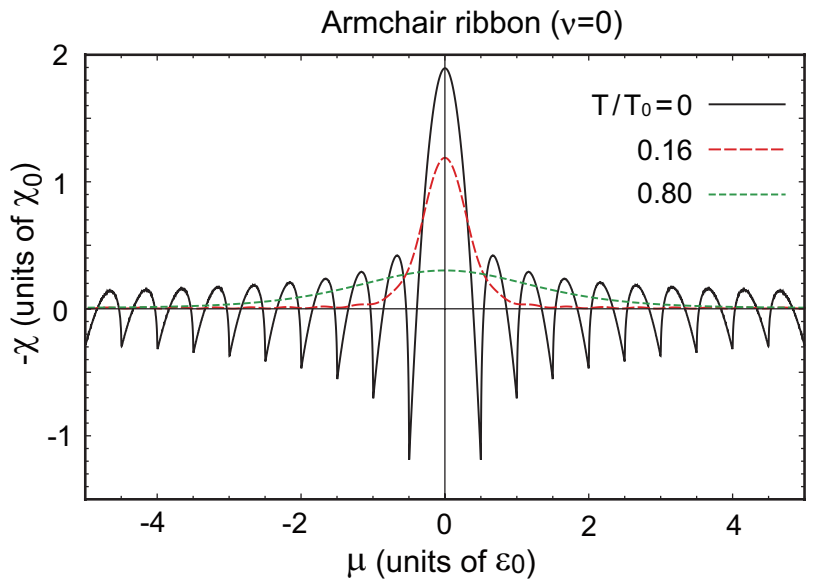

FIG. 4: Magnetic susceptibility against chemical potential $\mu$ for metallic armchair ribbon $(\nu=0)$ at several different temperatures.

\section{NUMERICAL RESULTS}

\section{A. Magnetic susceptibility}

Lower panels of Fig.3 show the orbital susceptibility $\chi\left(\varepsilon_{F}\right)$ of (a) zigzag, (b) metallic armchair $(\nu=0)$ and (c) semiconducting armchair $(\nu= \pm 1)$ ribbons at zero temperature, where the upward direction represents the negative (i.e., diamagnetic) susceptibility. The figures are to be compared with the band structures in upper panels. In every case, the magnitude of $\chi$ becomes the maximum at $\varepsilon_{F}=0$, and oscillates as a function of $\varepsilon_{F}$ in accordance with the subband structure. In the positive energy region, for example, the curve sharply rises when a subband starts to be occupied by electrons, while it tends to decrease otherwise. In large $\left|\varepsilon_{F}\right|$, the amplitude of the oscillation slowly attenuates approximately in proportional to $1 / \sqrt{\left|\varepsilon_{F}\right|}$.

The oscillating feature can be understood in terms of the band energy shift in an infinitesimal magnetic field. In the upper figures of Fig. 3, we plot as broken curves the energy band in some small $B$ calculated by the second order perturbation. Here the amplitude $B$ is set to some finite value for illustrative purpose. Generally the system is diamagnetic when the total energy shift caused by $B$ is positive, and paramagnetic when negative. In the metal- 


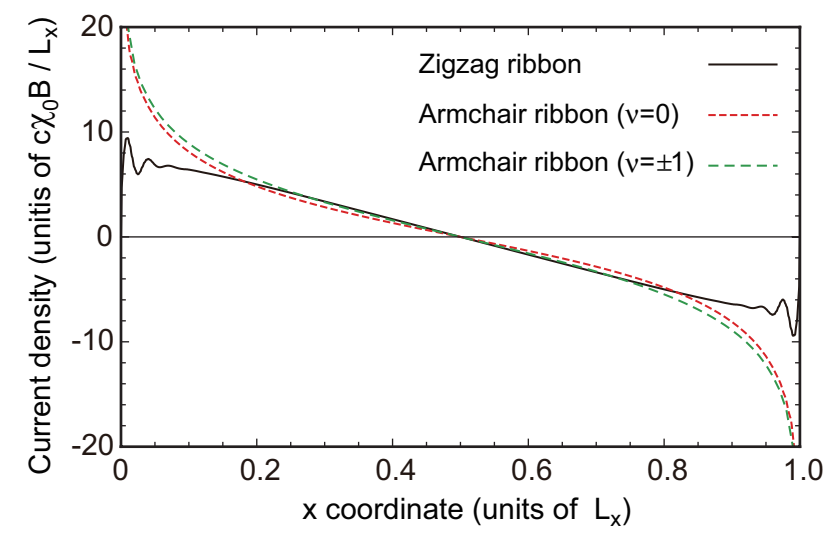

FIG. 5: Diamagnetic current density $j_{y}(x)$ of different types of graphene ribbons with $\varepsilon_{F}=0$ at $T=0$.

lic armchair ribbon (b), for example, we see that a pair of the first subbands $(n=0, s= \pm)$ shift towards zero energy, due to the level repulsions from excited subbands nearby. All other bands $(n>0)$ move in the opposite direction away from zero energy, while the absolute shifts are much smaller than that of $n=0$.

When $\varepsilon_{F}$ is zero, the energy gain of the first valence band $(s=-, n=0)$ exceeds the energy loss of all other valence bands $(s=-,|n| \geq 1)$, resulting in the total diamagnetism. When $\varepsilon_{F}$ is shifted to positive side, the diamagnetism decreases because the first conduction band $(s=+, n=0)$ has a negative shift and gives paramagnetism. When the second conduction band $(s=+,|n|=1)$ starts to be filled, the susceptibility suddenly jumps to diamagnetic direction, because the shift is positive there and also the density of states diverges at the band bottom. The oscillation of other types, (a) and (c), can be explained in a similar manner.

In Fig. 4, the susceptibility at several different temperatures is plotted as a function of the chemical potential $\mu$. We here choose the metallic armchair ribbon $(\nu=0)$ while the qualitative property is the same in other cases. We define the characteristic temperature scale $T_{0}$ as

$$
k_{B} T_{0}=\varepsilon_{0},
$$

at which the thermal broadening energy is of the order of the subband interval energy. We see that the oscillation rapidly disappears once $T$ becomes of the order of $T_{0}$, leaving only single diamagnetic peak at $\varepsilon_{F}=0$. When $T>\sim T_{0}$, the curve becomes almost identical with the bulk susceptibility, Eq. (29). We also confirmed that the integration of $\chi$ over $\varepsilon_{F}$ is identical with the bulk value $-g_{v} g_{s} e^{2} v^{2} /\left(6 \pi c^{2}\right)$ within the numerical accuracy, for all the types of ribbons considered here. This fact suggests that the finite size effect disappears $T>\sim T_{0}$, regardless of the edge configuration.

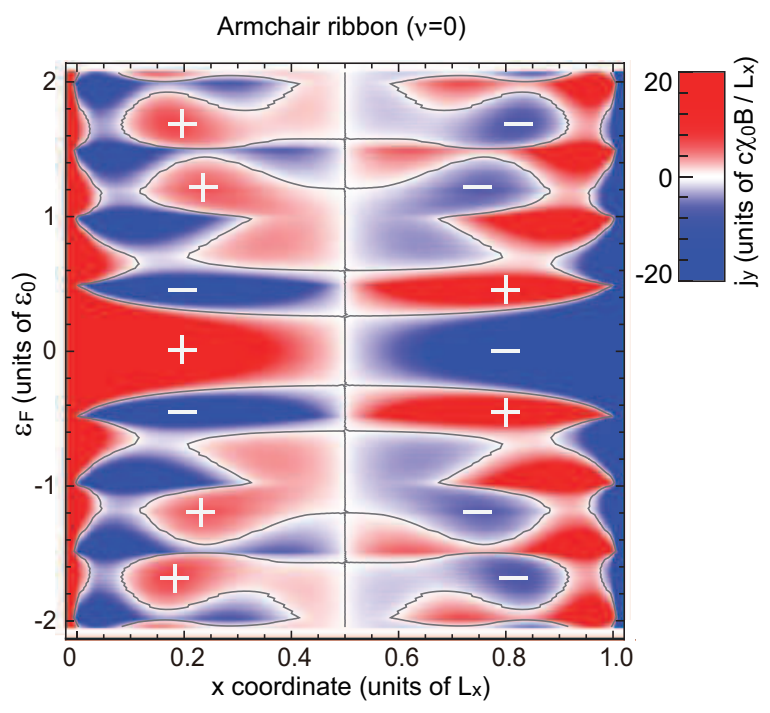

FIG. 6: Two-dimensional density plot $j_{y}\left(x ; \varepsilon_{F}\right)$ of the diamagnetic current density of metallic armchair ribbon $(\nu=0)$, as a function of position $x$ (horizontal axis) and Fermi energy (vertical).

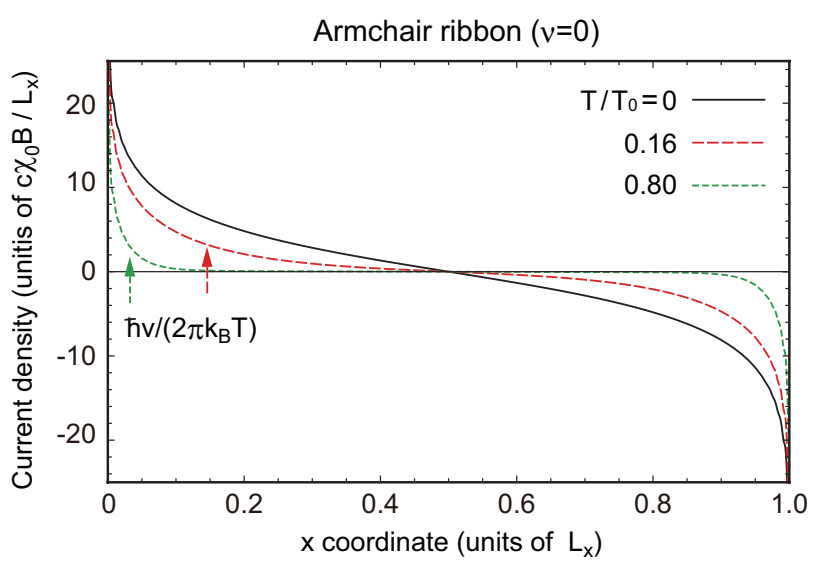

FIG. 7: Diamagnetic current density $j_{y}(x)$ of metallic armchair ribbon $(\nu=0)$ with $\varepsilon_{F}=0$, at several different temperatures. The vertical arrows indicate the characteristic length scale $\hbar v /\left(2 \pi k_{B} T\right)$ measured from $x=0$.

\section{B. Diamagnetic current density}

Fig. 5 shows the diamagnetic current density $j_{y}(x)$ in different types of graphene ribbons at $\varepsilon_{F}=0$ and $T=0$, calculated in the first order perturbation of $B$. The unit of current density is taken as $c \chi_{0} B / L_{x}$. The current flows in opposite directions in the left-hand side and right-hand side of the ribbon, to make a magnetization perpendicular to the layer. Reflecting the absence of the characteristic length scale, the current distribution is not localized to the edge but spread in the entire width in a form of slowly-varying monotonic function. 
In zigzag ribbons, the current density actually becomes absolute zero at $x=0$ and $L_{x}$, in accordance with the constraint argued in the previous section. The current sharply drops to zero at the edges, and some oscillatory feature remains around the edge due to a finite cut-off energy. When we increase the energy cut-off (not shown), the curve appears to slowly approach a fixed curve having a discontinuous jump at the edges. In the armchair ribbons, $j_{y}$ is not necessarily zero but logarithmically diverges at the both edges. The numerical calculation converges much more rapidly there, since there is no discontinuity as in the zigzag case.

Fig. 6 is the two-dimensional density plot $j_{y}\left(x ; \varepsilon_{F}\right)$ of the diamagnetic current density of metallic armchair ribbon, as a function of position $x$ (horizontal axis) and Fermi energy (vertical). In increasing $\varepsilon_{F}$, the current distribution begins to oscillate as a function of $x$, with a characteristic wave length of the order of $k_{F}=\varepsilon_{F} /(\hbar v)$.

The temperature dependence of the current density at $\varepsilon_{F}=0$ is shown in Fig.7 for the same metallic armchair ribbon. When $T$ becomes as large as $T_{0}$, the current distribution is localized at the boundary forming the counter edge currents. This is the same temperature range where the oscillation of $\chi$ disappears and the bulk limit is achieved. The depth of the current distribution is characterized by

$$
\lambda_{\text {edge }}(T)=\frac{\hbar v}{2 \pi k_{B} T},
$$

which shrinks in increasing temperature. With the band velocity of graphene, $v \approx 10^{6} \mathrm{~m} / \mathrm{s}$, it is estimated as $\lambda_{\text {edge }} \approx[1 / T(K)] \mu \mathrm{m}$.

This behavior is intuitively explained using the plot of $j_{y}\left(x ; \varepsilon_{F}\right)$ in Fig.6. The current density at a finite $T$ is obtained by integrating $j_{y}\left(x ; \varepsilon_{F}\right)$ in $\varepsilon_{F}$ with the thermal averaging factor $-\partial f / \partial \varepsilon$. The current of the middle part of the ribbon vanishes in averaging the oscillating function in $\varepsilon_{F}$, while the cancellation is not complete only near the edges, since $j_{y}$ is always positive and negative in left and right ends, respectively. The similar temperature dependence of the current distribution is found in other types of ribbons considered here. This suggests that, in any finite pieces of graphene with length scale $L$, the finite-size effect disappears when $T>T_{0}$, and then the diamagnetic current circulates only near edge with a depth $\lambda_{\text {edge }}$.

\section{Relation to spin paramagnetism}

We neglect the effect of the electron spin through out the present analysis. In a zigzag ribbon, particularly, the large density of states contributed by the zero-energy flat band is expected to give a significant magnitude of Pauli paramagnetism and reduce the orbital diamagnetism. ${ }^{31}$

The ratio between two effects can be quantitatively estimated as follows. The susceptibility of Pauli param- agnetism is given by

$$
\chi_{\text {para }}=\left(\frac{g}{2}\right) \mu_{B}^{2} D(\varepsilon),
$$

where $g \sim 2$ is the $g$-factor for graphene electron, $\mu_{B}=$ $e \hbar /(2 m c)$ is the Bohr-magneton with $m$ being the freeelectron mass, and $D(\varepsilon)$ is the density of states per area given by the zero-energy flat band. Since the number of edge states accommodated in a ribbon of the length $L$ is $\sim L / a$ per spin and per valley, ${ }^{31}$ we have

$$
D(\varepsilon) \sim \frac{g_{v} g_{s}}{L^{2}} \frac{L}{a} \delta(\varepsilon),
$$

which gives a delta-function singularity in $\chi_{\text {para }}$.

By comparing $\chi_{\text {para }}$ with the bulk orbital diamagnetism $\chi_{\text {dia }}$, Eq. $(28)$, we obtain

$$
\left|\frac{\chi_{\text {para }}}{\chi_{\text {dia }}}\right|=\frac{3 \pi}{2} \frac{\hbar^{2}}{m^{2} v^{2} a L} \sim 1.0 \times \frac{a}{L},
$$

which is negligible in a wide strip with $L \gg a$. In a low temperature such that $k_{B} T<\sim \varepsilon_{0}$, however, $\chi_{\text {dia }}$ cannot be regarded as thermally broadened delta-function due to the effect of the subband formation, and then $\chi_{\text {para }}$ overcomes $\chi_{\text {dia }}$ only at $\varepsilon_{F}=0$.

\section{CARBON NANOTUBES}

The carbon nanotube is a quasi-one-dimensional system similar to graphene ribbon, but different in that there are no edges. ${ }^{42,43}$ Experimentally, graphene nanoribbons with smooth edges can be obtained by unzipping the carbon nanotubes, i.e., lengthwise cutting of carbon nanotube side walls. ${ }^{40,41}$ Then we may ask which of the ribbon and the original nanotube has greater diamagnetism, and how the susceptibility oscillation in $\varepsilon_{F}$ changes in unzipping. The orbital susceptibility of carbon nanotube was theoretically studied for small Fermi energies in the effective mass approximation ${ }^{23,24}$. Here we compute full Fermi energy dependence in parallel fashion to the analysis for ribbons.

A carbon nanotube is characterized by a chiral vector,

$$
\boldsymbol{L}=n_{a} \boldsymbol{a}+n_{b} \boldsymbol{b},
$$

where the atom at $\boldsymbol{L}$ on a graphene sheet is rolled up onto the origin in constructing a tube. The boundary condition is given by $\psi_{\mathrm{A}}\left(\boldsymbol{R}_{\mathrm{A}}\right)=\psi_{\mathrm{A}}\left(\boldsymbol{R}_{\mathrm{A}}+\boldsymbol{L}\right)$ and $\psi_{\mathrm{B}}\left(\boldsymbol{R}_{\mathrm{B}}\right)=\psi_{\mathrm{B}}\left(\boldsymbol{R}_{\mathrm{B}}+\boldsymbol{L}\right)$. For the effective mass wavefunction, it is written as ${ }^{53}$

$$
\begin{gathered}
\boldsymbol{F}^{\mathrm{K}}(\boldsymbol{r}+\boldsymbol{L})=\exp \left(-\frac{2 \pi i}{3} \nu\right) \boldsymbol{F}^{\mathrm{K}}(\boldsymbol{r}), \\
\boldsymbol{F}^{\mathrm{K}^{\prime}}(\boldsymbol{r}+\boldsymbol{L})=\exp \left(+\frac{2 \pi i}{3} \nu\right) \boldsymbol{F}^{\mathrm{K}^{\prime}}(\boldsymbol{r}) .
\end{gathered}
$$

Here $\boldsymbol{F}^{\mathrm{K}}=\left(F_{\mathrm{A}}^{\mathrm{K}}, F_{\mathrm{B}}^{\mathrm{K}}\right)$ etc., and $\nu$ is an integer $(0, \pm 1)$ defined by

$$
n_{a}+n_{b}=3 m+\nu,
$$


with integer $m$.

For $K$ point, the eigenstates are immediately obtained as

$$
\begin{aligned}
& \boldsymbol{F}_{s n k_{y}}^{\mathrm{K}}(\boldsymbol{r})=\frac{e^{i k_{y} y}}{\sqrt{2 L_{x} L_{y}}}\left(\begin{array}{c}
e^{i k_{n} x} \\
s e^{i\left(k_{n} x+\theta\right)} \\
0 \\
0
\end{array}\right), \\
& \varepsilon_{s n k_{y}}=s \hbar v \sqrt{k_{y}^{2}+k_{n}^{2}}
\end{aligned}
$$

where

$$
k_{n} \equiv \frac{2 \pi}{L_{x}}\left(n-\frac{\nu}{3}\right), n=0, \pm 1, \pm 2, \cdots,
$$

and $L_{x}=|\boldsymbol{L}|$ and $L_{y}$ is length of the carbon nanotube. The system is metallic when $\nu=0$, and semiconducting when $\nu= \pm 1$. The band structure looks similar to armchair graphene ribbon's, but the unit of momentum quantization doubled compared to Eq. (15), leading to wider energy spacing between subbands. The energy band for $\mathrm{K}^{\prime}$ is obtained by replacing $k_{y}$ by $-k_{y}$ and also $\nu$ by $-\nu$.

When a uniform magnetic field $B$ is applied perpendicularly to the nanotube axis, the vector potential can be taken as

$$
\boldsymbol{A}(\boldsymbol{r})=\left(0, \frac{B L_{x}}{2 \pi} \sin \frac{2 \pi x}{L_{x}}\right) .
$$

We should note that the expression differs from that for ribbon, Eq. (18), because the magnetic field perpendicular to the tube surface is not a constant, but a sinusoidal function in $x$. Except for that, the magnetic susceptibility $\chi_{\text {tube }}\left(\varepsilon_{F}\right)$ is calculated in the same formula, Eq. (26).

The susceptibility of the carbon nanotube is naturally related to that of graphene against a spatial varying magnetic field $B(q) \sin q x$ with $q=2 \pi / L_{x} \equiv q_{0}$. When we define the $q$-dependent susceptibility of graphene as $\chi_{\mathrm{gr}}(q) \equiv m(q) / B(q),{ }^{16}$ we obtain a relation

$$
\left\langle\chi_{\text {tube }}\left(\varepsilon_{F}\right)\right\rangle_{\varphi}=\frac{1}{2} \chi_{\mathrm{gr}}\left(q_{0} ; \varepsilon_{F}\right) .
$$

Here \langle\rangle$_{\varphi}$ represents an average over a phase factor $\varphi$ which twists the boundary condition of carbon nanotube as $\psi(\boldsymbol{r}+\boldsymbol{L})=\exp (2 \pi i \varphi) \psi(\boldsymbol{r})$. Physically, the phase factor corresponds to threading a magnetic flux of $(h / e) \varphi$ into the nanotube cross section. ${ }^{23,53}$ It changes momentum quantization of Eq. (40) to $k_{n}=\left(2 \pi / L_{x}\right)(n+\varphi-$ $\nu / 3$ ), and the flux averaging over $\varphi$ smears the difference in $\nu$. The factor $1 / 2$ in Eq. (42) enters because the average of the squared magnetic field on the nanotube surface is $B\left(q_{0}\right)^{2} / 2$.

At the zero temperature, $\chi_{\mathrm{gr}}\left(q ; \varepsilon_{F}\right)$ is explicitly evaluated as ${ }^{16}$

$$
\begin{aligned}
& \chi_{\mathrm{gr}}\left(q ; \varepsilon_{F}\right)=-\frac{g_{v} g_{s} e^{2} v}{16 \hbar c^{2}} \frac{1}{q} \theta\left(q-2 k_{F}\right) \\
& \times\left[1+\frac{2}{\pi} \frac{2 k_{F}}{q} \sqrt{1-\left(\frac{2 k_{F}}{q}\right)^{2}}-\frac{2}{\pi} \sin ^{-1} \frac{2 k_{F}}{q}\right],
\end{aligned}
$$

where $k_{F}=\left|\varepsilon_{F}\right| /(\hbar v)$ is the Fermi wave number and $\theta(x)$ is defined by $\theta(x)=1(x>0)$ and $0(x<0)$. Using Eqs. (42) and (43), the flux-averaged susceptibility integrate is shown to be

$$
\left\langle\int_{-\infty}^{\infty} \chi_{\text {tube }}\left(\varepsilon_{F}\right) d \varepsilon_{F}\right\rangle_{\varphi}=\frac{1}{2}\left(-g_{v} g_{s} \frac{e^{2} v^{2}}{6 \pi c^{2}}\right),
$$

which is exactly half of graphene's, suggesting that the susceptibility is effectively smaller in nanotube than in ribbon. This is simply because the $B$-field component penetrating the lattice plane is smaller in the nanotube due to its cylindrical shape.

The susceptibility before taking flux average can be calculated in numerics. Fig. 8 (a) shows $\chi_{\text {tube }}\left(\varepsilon_{F}\right)$ for the metallic $(\nu=0)$ and the semiconducting $(\nu= \pm 1)$ nanotubes, together with the flux average. It has an oscillatory behavior similar to the graphene ribbon's, while $\chi$ in $\left|\varepsilon_{F}\right|>\hbar v q / 2$ completely vanishes after flux average. ${ }^{16}$ In increasing temperature (not shown), the oscillation immediately disappears, leaving a single peak regardless of $\nu$, similar to the graphene ribbon.

Fig. 8 (a) compares the susceptibility of a carbon nanotube and that of corresponding graphene ribbon unzipped from the same nanotube. Here we chose a zigzag ribbon as an example, when the corresponding nanotube becomes an armchair nanotube which is always metal-

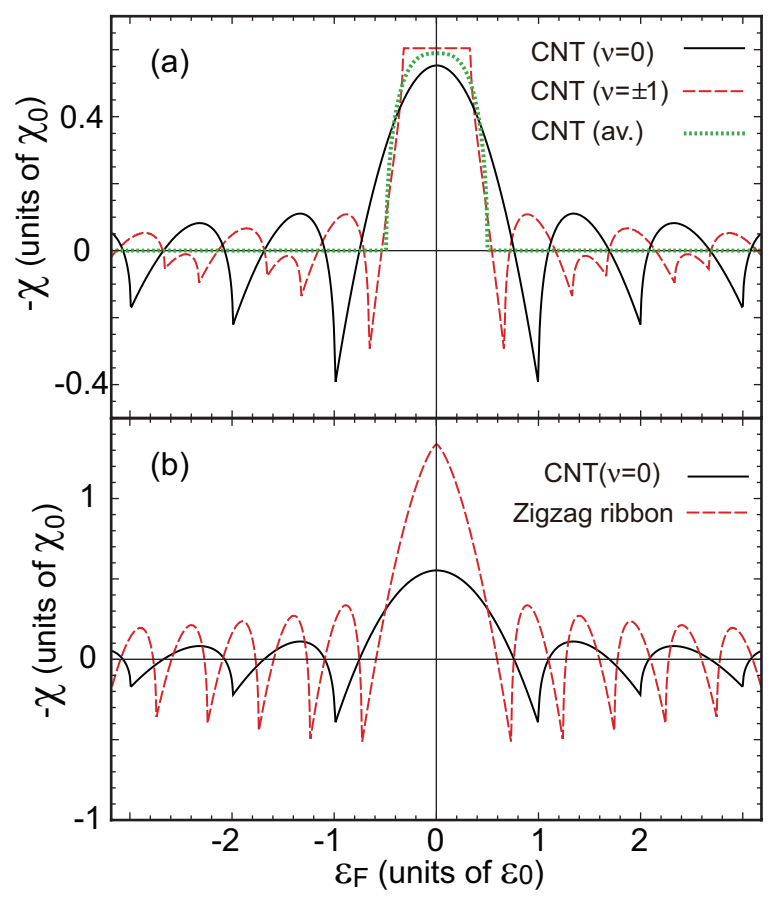

FIG. 8: (a) Magnetic susceptibility of carbon nanotubes. Solid (black), dashed (red) and dotted (green) curves are for metallic $(\nu=0)$, semiconducting $(\nu= \pm 1)$, and the flux average, respectively. (b) Magnetic susceptibility of the carbon nanotube of $\nu=0$ (solid black) and the zigzag ribbon unzipped from the same nanotube (dashed red). 
lic $(\nu=0) .{ }^{53}$ The oscillation period of the nanotube is approximately twice as large as that of the ribbon, reflecting the wider subband spacing. Overall magnitude of $\chi$ is smaller in nanotube roughly by factor 2 . The integrate of susceptibility in $\varepsilon_{F}$ differs in factor 2 in numerical accuracy, in accordance with the above arguments.

\section{RANDOMLY-STACKED MULTILAYER GRAPHENE}

The diamagnetism can be made even greater by stacking graphenes in three dimensions. The recent experimental technique realizes a novel kind of graphene multilayer in which successive layers are stacked with random rotating angles. ${ }^{45-47}$ There it is known that the interlayer coupling is significantly weakened and the Dirac cone is kept almost intact near zero energy as long as the rotating angle is not too small. ${ }^{48-51}$ The orbital susceptibility of such a system is expected to be much stronger than graphite in which the delta-function peak of $\chi\left(\varepsilon_{F}\right)$ is much broadened and shortened by the regular interlayer coupling. ${ }^{18,19}$

Here we consider the orbital diamagnetism of a finitesized piece of random-stacked graphene multilayers. In calculations, we self-consistently include the effect of the counter magnetic field induced by the diamagnetic current itself. This is essential because, as we will show in the following, the counter magnetic field of this system can be of the same order of the external magnetic field, and even nearly perfect screening is possible in low temperatures.

For simplicity, we completely neglect the interlayer coupling and regard the system as a set of independent single layer graphenes. We also assume that each layer has the identical shape with a characteristic length scale $L$, and that the system is large enough that the thermal broadening energy $k_{B} T$ is much larger than $2 \pi \hbar v / L$. According to the previous discussions, we then expect that the susceptibility of each layer is given by the bulk limit $\chi_{\text {gr }}$ in Eq. (29), and also the depth of the edge current $\lambda_{\text {edge }}$ of Eq. (32) can be neglected with respect to the system size $L$.

Let us consider a situation where a external field $B_{\text {ext }}$ is applied perpendicularly to graphene plane of the random stacked multilayer. The total magnetic field $B$ penetrating the system is

$$
B=B_{\text {ext }}+\Delta B,
$$

where $\Delta B$ is the counter field caused by graphene electrons. The total field $B$ induces the magnetism in each layer, $M=\chi_{\mathrm{gr}} B S$, with $S$ being the area of the layer. This is related to the diamagnetic edge current $I$ of each single layer by

$$
I=\frac{c M}{S}=c \chi_{\mathrm{gr}} B
$$

Since the ring current $I$ exists every interlayer distance $d$, it induces a counter-magnetic field inside the system as

$$
\Delta B=\frac{4 \pi}{c} \frac{I}{d} .
$$

Solving the set of equations, we find that the dimensionless volume susceptibility becomes

$$
\chi_{3 \mathrm{D}} \equiv \frac{\Delta B}{B_{\mathrm{ext}}}=\frac{-1}{1-d /\left(4 \pi \chi_{\mathrm{gr}}\right)} .
$$

At the charge neutral point $\mu=0$, in particular, we have

$$
\chi_{3 \mathrm{D}}(\mu=0)=\frac{-1}{1+k_{B} T / \Delta},
$$

where $\Delta$ is a characteristic energy scale defined by

$$
\Delta=\frac{g_{v} g_{s}}{6}\left(\frac{v}{c}\right)^{2} \frac{e^{2}}{d} \approx 0.03 \mathrm{meV},
$$

and $d$ is assumed to be the interlayer spacing of graphite, $0.334 \mathrm{~nm}$.

In decreasing the temperature, $\chi_{3 \mathrm{D}}$ monotonically increases in the negative direction, and approaches -1 , where the prefect magnetic field screening is achieved. This reflects the property of the single-layer susceptibility, Eq. (29), of which peak value at Dirac point diverges in $T \rightarrow 0$. In contrast, $\chi_{3 \mathrm{D}}$ of the graphite is of the order of $10^{-4}$ and is not much enhanced in low temperatures ${ }^{57}$ because $\chi\left(\varepsilon_{F}\right)$ is already broadened by the interlayer coupling energy about $4000 \mathrm{~K} .{ }^{18,19} \mathrm{~A}$ three-dimensional bulk material composed of random-stacked graphenes, if realized, would be the strongest diamagnetic material than any other known substances except for the superconductors. Including the effect of the residual interlayer coupling between misoriented layers may set the upper limit to $\chi_{3 \mathrm{D}}$, while we leave the detailed analysis for a future problem.

\section{CONCLUSION}

We have studied the orbital diamagnetism of graphene ribbons using the effective-mass approximation, to figure out its dependence on temperature and Fermi energy, and also the finite-size effect on the delta-function singularity in the bulk limit. In increasing temperature, an oscillatory behavior in the orbital susceptibility $\chi\left(\varepsilon_{F}\right)$ is eventually smeared out, approaching the bulk limit i.e., a thermally broadened delta-function centered at $\varepsilon_{F}=0$. The electric current responsible for the diamagnetism spreads entirely in the sample at $T=0$ reflecting the absence of the characteristic wavelength, while as $T$ is increased, the current density tends to localize near the edge with a depth $\sim \hbar v /\left(2 \pi k_{B} T\right)$.

We also see a carbon nanotube, another form of quasione-dimensional carbon, exhibits a similar oscillation in 
$\chi\left(\varepsilon_{F}\right)$, but the overall magnitude is reduced by a factor 2 compared to the corresponding ribbon having the same width. The result is applied to estimate the threedimensional bulk susceptibility of random-stacked multilayer graphene. There we showed that the external magnetic field is significantly screened inside the sample.

\section{ACKNOWLEDGMENTS}

This project has been funded by JST-EPSRC JapanUK Cooperative Programme Grant No. EP/H025804/1.
1 J. W. McClure, Phys. Rev. 104, 666 (1956).

2 J. W. McClure, Phys. Rev. 119, 606 (1960).

3 M. P. Sharma, L. G. Johnson, and J. W. McClure, Phys. Rev. B 9, 2467 (1974).

${ }^{4}$ K. S. Novoselov, A. K. Geim, S. V. Morozov, D. Jiang, Y. Zhang, S. V. Dubonos, I. V. Grigorieva, and A. A. Firsov, Science 306, 666 (2004).

${ }^{5}$ K. S. Novoselov, A. K. Geim, S. V. Morozov, D. Jiang, M. I. Katsnelson, I. V. Grigorieva, S. V. Dubonos, and A. A. Firsov, Nature 438, 197 (2005).

6 Y. Zhang, Y.-W. Tan, H. L. Stormer, and P. Kim, Nature 438, 201 (2005).

7 J. C. Slonczewski and P. R. Weiss, Phys. Rev. 109, 272 (1958).

8 D. P. DiVincenzo and E. J. Mele, Phys. Rev. B 29, 1685 (1984).

${ }^{9}$ G. W. Semenoff, Phys. Rev. Lett. 53, 2449 (1984).

10 S. G. Sharapov, V. P. Gusynin, and H. Beck, Phys. Rev. B 69, 075104 (2004).

11 H. Fukuyama, J. Phys. Soc. Jpn. 76, 043711 (2007).

12 M. Nakamura, Phys. Rev. B 76, 113301 (2007).

13 M. Koshino and T. Ando, Phys. Rev. B 75, 235333 (2007).

14 A. Ghosal, P. Goswami, and S. Chakravarty, Phys. Rev. B 75, 115123 (2007).

15 T. Ando, Physica E 40, 213 (2007).

16 M. Koshino, Y. Arimura, and T. Ando, Phys. Rev. Lett. 102, 177203 (2009).

17 M. Koshino and T. Ando, Phys. Rev. B 81, 195431 (2010).

18 S. A. Safran, Phys. Rev. B 30, 421 (1984).

19 M. Koshino and T. Ando, Phys. Rev. B 76, 085425 (2007).

20 S. A. Safran and F. J. DiSalvo, Phys. Rev. B 20, 4889 (1979).

21 J. Blinowski and C. Rigaux, J. Phys. (Paris) 45, 545 (1984).

22 R. Saito and H. Kamimura, Phys. Rev. B 33, 7218 (1986).

${ }^{23}$ H. Ajiki and T. Ando, J. Phys. Soc. Jpn. 62, 2470 (1993); J. Phys. Soc. Jpn. 63, 4267 (1994) (Erratum).

${ }^{24}$ H. Ajiki and T. Ando, J. Phys. Soc. Jpn. 64, 4382 (1995).

25 M. Yamamoto, M. Koshino, and T. Ando, J. Phys. Soc. Jpn. 77, 084705 (2008).

26 M. Nakamura and L. Hirasawa, Phys. Rev. B 77, 045429 (2008).

27 A. H. Castro Neto, F. Guinea, N. M. Peres, K. S. Novoselov, and A. K. Geim, Rev. Mod. Phys. 81, 109 (2009).

28 A. Kobayashi, Y. Suzumura, and H. Fukuyama, J. Phys. Soc. Jpn. 77, 064718 (2008). Hall effect and orbital diamagnetism in zerogap state of molecular conductor $\alpha$ $(\mathrm{BEDT}-\mathrm{TTF})_{2} \mathrm{I}_{3}$

29 M. Fujita, K. Wakabayashi, K. Nakada, and K. Kusakabe, J. Phys. Soc. Jpn. 65, 1920 (1996).

${ }^{30}$ K. Nakada, M. Fujita, G. Dresselhaus, and M. S. Dressel- haus, Phys. Rev. B 54, 17954 (1996).

31 K. Wakabayashi, M. Fujita, H. Ajiki, and M. Sigrist, Phys. Rev. B59, 8271 (1999).

32 M. Ezawa, Phys. Rev. B 73, 045432 (2006).

33 L. Brey, and H. Fertig, Phys. Rev. B 73, 195408 (2006).

${ }^{34}$ L. Brey, and H. Fertig, Phys. Rev. B 73, 235411 (2006).

35 Y-W. Son, M. L. Cohen, S. G. Louie, Phys. Rev. Lett. 97, 216803 (2006).

36 Y-W. Son, M. L. Cohen, S. G. Louie, Nature 444, 347 (2006).

37 M. Y. Han, B. Özyilmaz, Y. Zhang, and P. Kim, Phys. Rev. Lett. 98, 206805 (2007).

38 Z. Chen, Y.-M. Lin, M. J. Rooks, and P. Avouris, Physica E 40, 228 (2007).

39 X. Li, X. Wang, L. Zhang, S. Lee, and H. Dai, Science 319, 1229 (2008).

40 D. V. Kosynkin, A. L. Higginbotham, A. Sinitskii, J. R. Lomeda, A. Dimiev, B. K. Price, J. M. Tour, Nature 458, 872 (2009).

${ }^{41}$ L. Y. Jiao, L. Zhang, X. R. Wang, G. Diankov, H. J. Dai, Nature 458, 877 (2009).

42 S. Iijima, Nature (London) 354, 56 (1991).

43 S. Iijima, T. Ichihashi and Y. Ando, Nature 356, 776 (1992).

44 J. Liu, Z. Ma, A. R. Wright, and and Chao Zhang, J. Appl. Phys. 103, 103711 (2008).

45 C. Berger, Z. Song, X. Li, X. Wu, N. Brown, C. Naud, D. Mayou, T. Li, J. Hass, A. N. Marchenkov, E. H. Conrad, P. N. First, and W. A. de Heer, Science 312, 1191 (2006).

46 J. Hass, R. Feng, J. E. Millan-Otoya, X. Li, M. Sprinkle, P. N. First, W. A. de Heer, E. H. Conrad, and C. Berger, Phys. Rev. B 75, 214109 (2007).

47 G. Li, A. Luican and E. Y. Andrei, Phys. Rev. Lett. 102, 176804 (2009).

48 J. M. B. Lopes dos Santos, N. M. R. Peres, A.H. Castro Neto, Phys. Rev. Lett. 99, 256802 (2007).

49 S. Latil, V. Meunier, and L. Henrard, Phys. Rev. B 76, 201402(R) (2007).

50 S. Shallcross, S. Sharma and O.A. Pankratov, Phys. Rev. Lett. 101, 056803 (2008).

51 E. J. Mele, Phys. Rev. B 81, 161405 (2010).

52 N. H. Shon and T. Ando, J. Phys. Soc. Jpn. 67, 2421 (1998).

53 T. Ando, J. Phys. Soc. Jpn. 74, 777 (2005).

54 V. P. Gusynin and S. G. Sharapov, Phys. Rev. Lett. 95, 146801 (2005).

55 N. M. R. Peres, F. Guinea, and A. H. Castro Neto, Phys. Rev. B 73, 125411 (2006).

56 P. Marconcini, and M. Macucci, Rivista Del Nuovo Cimento 34489 (2011).

57 K. S, Krishnan and N. Ganguli, Nature 139, 155 (1937). 